\title{
Solution stability and variability in a simple model of globular proteins
}

\author{
Richard P. Seara) \\ Department of Physics, University of Surrey, Guildford, Surrey GU2 7XH, United Kingdom
}

(Received 17 June 2003; accepted 15 October 2003)

\begin{abstract}
It is well known among molecular biologists that proteins with a common ancestor and that perform the same function in similar organisms, can have rather different amino-acid sequences. Mutations have altered the amino-acid sequences without affecting the function. A simple model of a protein in which the interactions are encoded by sequences of bits is introduced, and used to study how mutations can change these bits, and hence the interactions, while maintaining the stability of the protein solution. This stability is a simple minimal requirement on our model proteins which mimics part of the requirement on a real protein to be functional. The properties of our model protein, such as its second virial coefficient, are found to vary significantly from one model protein to another. It is suggested that this may also be the case for real proteins in vivo. (c) 2004 American Institute of Physics. [DOI: 10.1063/1.1631918]
\end{abstract}

\section{INTRODUCTION}

Proteins are linear heteropolymers: they are linear sequences of monomers, each of which is one of twenty different types. Different proteins have different sequences of amino acids. These differences allow proteins to perform the huge range of tasks they do in living cells. But this does not mean that two proteins that do the same job necessarily have the same sequence. For example, many organisms have enzymes called adenylate kinases which perform essentially the same job in the cytoplasm of each organism. But the amino acid sequences of adenylate kinases vary very widely, even though they are all doing the same job in more-or-less the same milieu. Below are the amino-acid sequences of the adenylate kinases of two prokaryotes. ${ }^{1}$ First that of Escherichia coli,

\section{MRI I LLGAPGAGKGTQAQF IMEKYGIPQISTGDMLRAAVKSGSELGKQAK DIMDAGKLVTDELVIALVKERIAQEDCRNGF LLDGFPRT IPQADAMKEAG INVDYVLEFDVPDELIVDRIVGRRVHAP SGRVYHVKF NP P KVEGKDDVTG EELTTRKDDQEETVRKRLVEYHQMTAP LIGYYSKEAEAGNTKYAKVDGTK PVAEVRADLEKILG,}

and second that of Vibrio cholerae,

MRI I LLGAP GAGKGTQAQF IMEKF GIPQISTGDMLRAA IKAGTELGKQAK AVIDAGQLVSDD I ILGLIKERIAQADCEKGF LLDGFPRT IPQADGLKEMG INVDYVIEFDVADDVIVERMAGRRAHLP SGRTYHVVYNPP KVEGKDDVTG EDLVIREDDKEETVRARLNVYHTQTAP LIEYYGKEAAAGKTQYLKFDGTK QVSEVSADIAKALA,

where the sequences are given as a sequence of the 1-letter codes for the amino acids of which they are made. The first amino acid is an M (methionine), the second is an R (arginine) and so on. The sequence is read as English text, from top left to bottom right. See any molecular biology or biochemistry textbook ${ }^{2-4}$ for an introduction to amino acids and proteins. Note that there are many differences between the sequences! The amino-acid sequences of proteins are very different while keeping the function. Also, we picked adenylate kinases only in order to have a concrete example; it is a

a)Electronic mail: r.sear@surrey.ac.uk general property of proteins. The function of adenylate kinases is irrelevant to our discussion of stability, beyond the fact that they function as enzymes as monomers in solution inside cells. Here we will concentrate entirely on globular proteins, the proteins that exist in solution not embedded in membranes.

Now, the simplest thing to do when faced with this radical difference in sequence without a corresponding difference in function is to ignore it. To assume that the two proteins interact and behave in a very similar manner. But do they? As they both function as proteins inside the cytoplasm of bacteria they are both clearly soluble and do not stick to 
things they should not stick to in vivo. However, this does not mean that their solubilities, for example, are necessarily equal. Both their solubilities are sufficient to allow them to function but one may exceed the minimum solubility by a large margin and one by a small margin. It would be of interest to know what these margins are and how they vary from protein to protein, not only because we wish to understand how proteins function and have evolved in vivo, but to help us process, purify, and crystallize proteins. If a protein is only marginally soluble in the conditions in vivo, then it may aggregate when its environment (salt concentrations, temperature, etc.) are altered. We would like to understand and to be able to predict, the variability of properties, such as the solubility, of proteins.

We will focus on the stability of solutions of proteins in their native state, i.e., we assume that the protein has folded into its native state and remains there. Thus we consider only folded proteins sticking together due to their surfaces attracting each other, not proteins partially unfolding and then aggregating due to the hydrophobic regions of the protein exposed by unfolding, attracting each other. So, our proteins will always be compact objects, more like colloidal particles than conventional polymers. This allows us to avoid the complex problem of protein (un)folding. Effectively, we assume that proteins such as the adenylate kinases of $E$. coli and $V$. cholerae differ only in their surfaces. Replacing one surface amino acid in the chain by another then changes only the surface and through that the protein-protein interaction. If a hydrophobic amino acid replaces a hydrophilic amino acid in a position on the chain where the chain is at the protein's surface, then we expect the surface to become more sticky, which would tend to decrease the second virial coefficient, whereas replacing a hydrophobic amino acid by a hydrophilic one should have the opposite effect. For simplicity, instead of having 20 different types of amino acids at the surface, we use a model whose surface is described by bits which have only two values: hydrophobic and hydrophilic. This is a rather gross approximation, the amino acids vary widely in size and some are charged, but we want the simplest possible model. The model is an extension of that considered in Ref. 5. A protein molecule is modeled by a cube, whose 6 faces interact with a short-ranged attraction, which is determined here by a sequence of $n_{B}$ bits. In Ref. 5 the interaction between faces was taken to be a random variable; we will discuss the differences between that model and the more complex one considered here in the conclusion. A schematic of the model is shown in Fig. 1.

We have talked of our model proteins being soluble in vivo. Real proteins have evolved to be so. The cytoplasm of bacteria such as $E$. coli and $V$. cholerae is very complex: bacteria typically have a few thousand different proteins, ${ }^{6}$ and any one of these proteins is then surrounded by thousands of different proteins, as well as RNA and DNA, small molecules such as nucleotides, etc. An individual enzyme must be soluble in the sense that it does not stick too strongly to not only other proteins of the same type but those of all the other types, as well as not binding to the RNA, DNA, etc. In future work, we will address this problem, but here we will keep things simple and consider only interactions be-

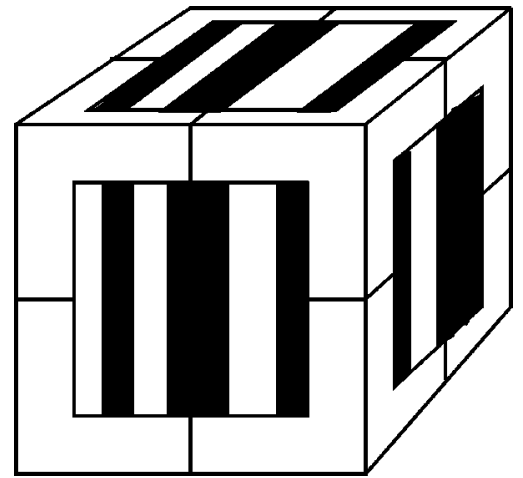

FIG. 1. A schematic of our model protein. It is drawn as a cube with the attractive patches drawn as striped patches on the faces of the cube. The model occupies $2 \times 2 \times 2=8$ lattice sites. The stripes represent the bits encoded in each patch of the model as a "bar code": if the black stripes are 1s and the white stripes are 0 s the front patch is 01011001 .

tween model proteins of one type. We will calculate the second virial coefficient only for the interaction of two model protein molecules of the same type. This is not realistic for an enzyme in a bacterial cell, as an individual enzyme will be present at rather low concentrations, even though the total protein concentration in bacterial cells is around $20 \%$ by volume. ${ }^{2,7}$ It is, however, a good place to start, and is realistic for a few exceptional cells, such as our red blood cells which contain very high concentrations of a single protein: hemoglobin. Future work will address this issue and will also look at proteins which bind to other proteins, as many proteins do.

The proteins whose sequences we gave in the first paragraph are presumably orthologs: they are both descended from a common ancestral protein but have evolved independently, keeping their function the same, since the $E$. coli and $V$. cholerae lineages separated. The fact that proteins with the same function, but that have evolved independently in different species, can have very different amino-acid sequences, is well known. Paralogs, proteins created by the duplication of a gene, also start with identical sequences but have sequences that diverge with time. The differences are believed to have arisen via random mutations which are not rejected by natural selection because they are not actively deleterious (to the survival of the organism) but also do not have any selective advantage. This theory of mutations changing the amino-acid sequences of proteins without improving or reducing its ability to function is called the theory of neutral evolution. ${ }^{8-10}$ The constraints placed on this neutral evolution by the requirement on the protein to fold have been considered, ${ }^{11-16}$ but not those due to the requirement of the protein to be soluble. The constraints placed on the sequences of RNA by the requirement to be functional and the evolution of these sequences, are analogous to the constraints on the sequences of, and evolution of proteins. They have been extensively studied and in many respects are rather better understood, essentially because RNA is simpler than protein. See the review of Higgs. ${ }^{17}$ However, there has been some work which has considered protein-to-protein variability, ${ }^{5,18}$ see also Ref. 19.

We will generate our model proteins at random (subject 


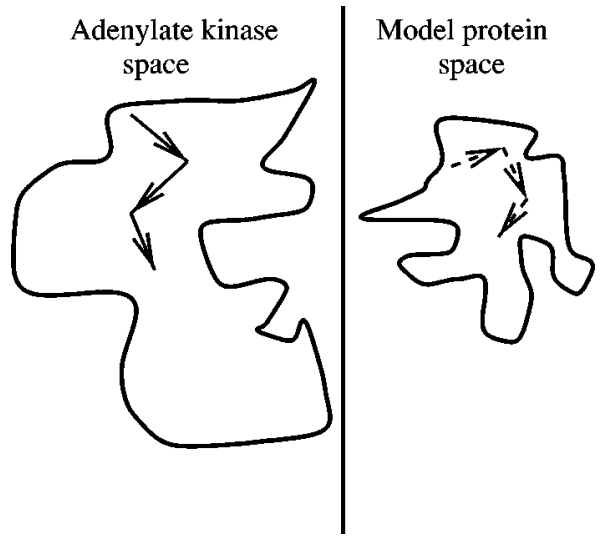

FIG. 2. A schematic of protein space for both a protein and a model protein. The arrows represent mutations changing a protein located at one point in protein space into a neighboring protein.

to the solubility constraint) and assume that the neutral evolution of proteins is close to a random walk from one sequence to another. This random walk occurs in what is often called "protein space," ${ }^{20}$ with each sequence a unique point in this space and two sequence neighbors if one of them can be transformed into the other by a single mutation. This protein space is vast. The set of soluble proteins exists in this protein space as a set of points, one for each soluble protein. A schematic of the protein spaces of proteins and model proteins is shown in Fig. 2. It is only very schematic, the space is huge and many dimensional. In each case the arrows represent a single mutation changing a protein into a neighboring protein. Below, we will generate random walks for our model proteins, and these will sample all soluble states with equal probability. When we come to applying our results to real, not model, proteins, we will have to assume that neutral evolution also samples proteins which are soluble with reasonably uniform probabilities.

In the following section, we will perform a simple analysis of sequence data, to look at variations in the number of hydrophobic amino acids. The model is defined in Sec. III, and the stability of its solutions estimated and discussed in Sec. IV. The last section is a conclusion.

\section{ANALYSIS OF SEQUENCE DATA}

The sequences of the adenylate kinases of $E$. coli and $V$. cholerae are both of viable enzymes, they are soluble in vivo and catalyze a reaction. Looking at them, an obvious question to ask is the following: How many sequences of amino acids are there, that fold up to form viable adenylate kinases? Both adenylate kinases have 214 amino acids. As there are 20 types of amino acids there are $20^{214} \simeq 10^{278}$ different amino acid sequences of 214 amino acids. An enormous number, of which presumably the vast majority do not fold into a unique native state, let alone are soluble and act as a catalyst. But it seems likely that the number of possible amino acid sequences that correspond to viable adenylate kinases is huge.

A database at SWISSPROT, ${ }^{21,22}$ called PROSITE, ${ }^{23,24}$ identified 152 amino-acid sequences as belonging to the adenylatekinase family of proteins (PROSITE accession number

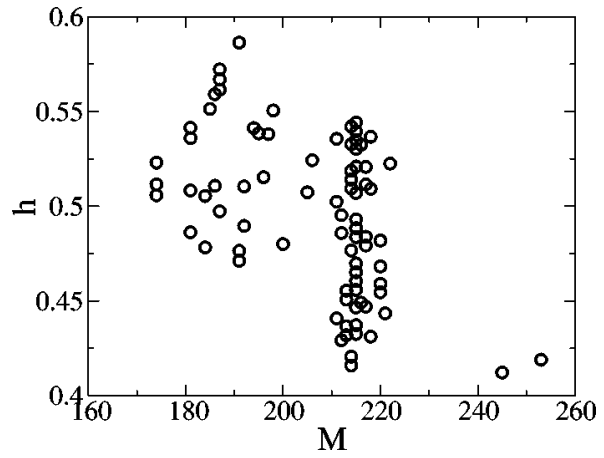

FIG. 3. A scatter plot of the fraction of its amino acids which are hydrophobic, $h$, vs the number of amino acids $M$. Results for the prokaryote members of the family of adenylate kinases are shown. The PROSITE accession number is PS00113.

PS00113). It did so by locating the amino acids of the active site of an adenylate kinase. ${ }^{23,24} 104$ of these sequences are from prokaryotes, of which we eliminate four sequences as they contain less than 100 amino acids and are presumably not complete proteins. This leaves 100 adenylate kinases; 2 of these kinases are the ones whose sequences are in the first paragraph. We can calculate the fraction of the amino acids of these adenylate kinases that are hydrophobic, $h$, and plot this against the length of the sequence, $M$ : the total number of amino acids in the sequence. The results are shown as a scatter plot, Fig. 3. The nine amino acids G, A, V, L, I, M, P, $\mathrm{F}$ and $\mathrm{W}$, are taken to be hydrophobic, and the remaining 11 to be hydrophilic. Here each amino acid is represented by its one-letter code: $\mathrm{G}$ for glycine, A for alanine, etc. The nine hydrophobic amino acids are those whose side chains are classified as nonpolar in Ref. 4 (Table 4-1, p. 58). There is some arbitrariness in where the dividing line is drawn between hydrophobic and hydrophilic amino acids, but different dividing lines give rather similar spreads in $h$.

For the present work, the key observation is that the fraction of an adenylate kinase's amino acids which are hydrophobic varies from protein to protein, as do other properties such as their net charge. ${ }^{18,25}$ In Sec. IV we will find that for our model, with a constraint imposed that model proteins are soluble, there is scatter in the fraction of its bits that are hydrophobic.

\section{MODEL}

The model is chosen to be as simple and as generic as possible, while having interactions which are mediated by surface patches whose interactions are a function of sequences or string of bits. The protein-protein interactions then depend on the values of these bits, some sets of values give proteins which strongly attract each other while other sets give proteins which largely repel each other. This is perhaps the simplest model of a globular protein which allows for mutations. Within the model these mutations flip one of the bits, a model of a mutation which converts a surface residue from a hydrophobic amino acid to a hydrophilic amino acid, or vice versa. A schematic of the model is shown in Fig. 1. An amino acid of a protein is called a residue. 


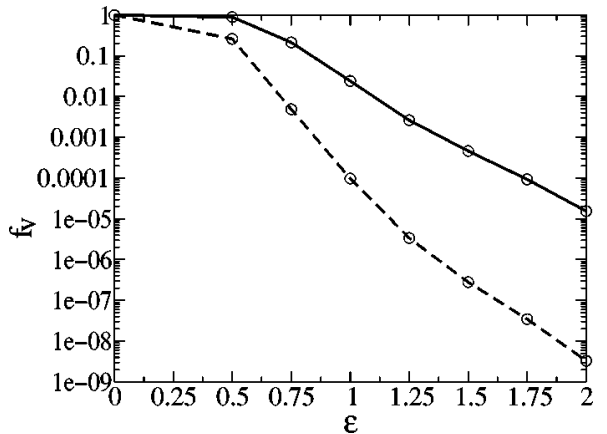

FIG. 4. A plot of the fraction of proteins with stable solutions, $f_{v}$, as a function of $\epsilon$. The solid and dashed curves are for $n_{B}=12$, and 18 bits, respectively.

The model protein is a cube, with each of its 6 faces having a single patch. ${ }^{5}$ The lattice is cubic and each protein occupies eight lattice sites arranged 2 by 2 by 2 ; see Fig. 1 . We make the model 2 sites across to reduce the range of the attraction, which is 1 site, to half the diameter of the hard core. The model proteins can rotate, and so have 24 distinct orientations. Each of the 6 faces of the cube has a patch, labeled $i=1$ to 6 , with patches 1 to 4 clockwise around a loop of 4 of the faces, and patches 5 and 6 on the remaining 2 faces. The interactions between model proteins are pairwise additive and consist of 2 parts. The first is simply an excluded-volume interaction: 2 proteins cannot overlap. The second is that if the faces of 2 proteins are in contact there is an energy of interaction between the 2 touching patches of the 2 proteins. By in contact we mean that the faces must overlap completely otherwise the energy of interaction is taken to be zero. Also, the model is such that the energy of interaction between two touching patches is a constant which does not change when the two proteins are rotated about the axis joining their centers. The touching patches are those on the faces of the 2 proteins that face each other. This is all as in Ref. 5, the difference is in how the interaction energy of a pair of patches $i$ and $j, u_{i j}$, is specified.

How a patch interacts is specified by a sequence or string of $n_{B}$ bits. If a bit has a value of 1 then the bit is hydrophilic or polar, whereas if it has a value of 0 then it is hydrophobic. The interaction energy of a pair of touching patches, $i$ and $j$, is then given by

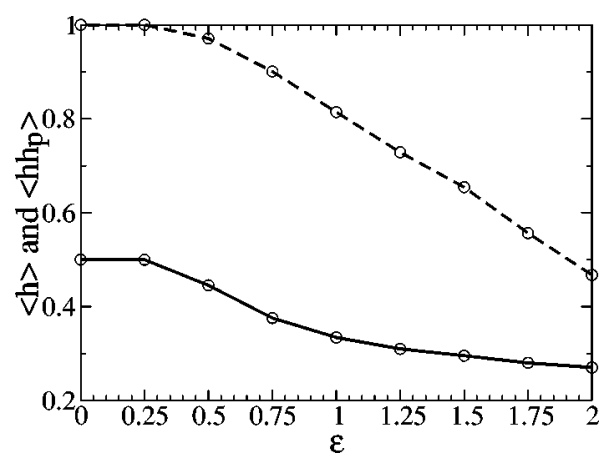

FIG. 5. A plot of the mean fraction of bits which are hydrophobic, $\langle h\rangle$, the solid curve, and of a measure of the correlation between a bit and the other bit it interacts with, $\left\langle h h_{p}\right\rangle$, the dashed curve.

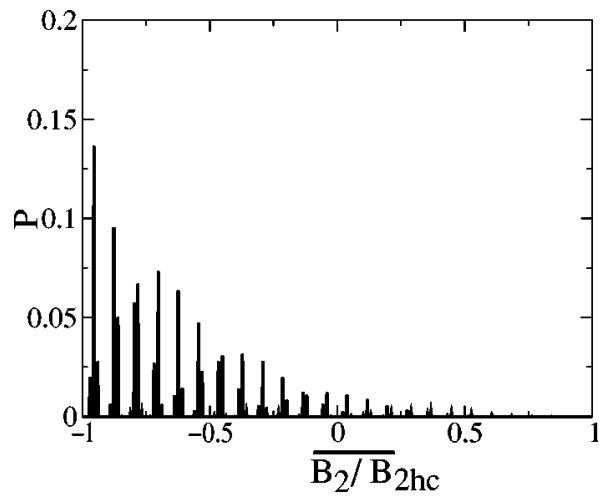

FIG. 6. The probability distribution function, $P$, for the reduced second virial coefficient, $B_{2} / B_{2 h c}$, for $n_{B}=18$ and $\epsilon=2$.

$$
u_{i j}=-\epsilon \sum_{\alpha=1}^{n_{B}}\left(b_{\alpha}^{(i)}-1\right)\left(b_{1+n_{B}-\alpha}^{(j)}-1\right)
$$

where $b_{\alpha}^{(i)}$ is bit number $\alpha$ of patch $i . \epsilon$ is the interaction energy of 2 hydrophobic bits. We use energy units such that the thermal energy $k_{\mathrm{B}} T=1$. Thus to calculate the interaction the string of bits of 1 of the patches is reversed and then the energy is just the sum of the number of pairs of corresponding bits where both bits are 0 , are hydrophobic. The only interaction is between 2 hydrophobic bits; there is no hydrophobic-hydrophilic or hydrophilic-hydrophilic interaction. The reason one of the strings is reversed is that if this is not done then the interaction between like patches, $j=i$, is just $\epsilon$ times the number of 0's in $i$ 's string. Reversing the strings removes this problem in a simple way. Of course, the interactions form a symmetric square matrix, $u_{i j}=u_{j i}$. Each of the 6 patches is taken to be labeled and so distinguishable, i.e., we take a pair of proteins where one protein can be obtained from the other by swapping a pair of the strings of bits, as 2 different proteins.

Thus, a protein is specified by giving values to the 6 strings of $n_{B}$ bits, and so there are $2^{6 n_{B}}$ possible different proteins. For all but rather small values of $n_{B}$, this is a very large number of possible proteins, e.g., for $n_{B}=18$, we have $3 \times 10^{32}$ different model proteins. This is, however, much smaller than the number of possible real proteins. Most of the calculations have been done for $n_{B}=18$, with a few for $n_{B}=12$, for comparison. We choose $n_{B}=18$ as being a sensible number as then the total number of bits which describe the surface is 108. Adenylate kinases, for example, have around 200 amino acids, of which about half are on the surface. Thus, we have about 1 bit per surface amino acid. Our model proteins can be thought of as existing in "protein space" with each possible protein represented by a point in this space, and each protein has $6 n_{B}$ neighbors, each of which is obtained by flipping 1 of the bits of the protein; see Fig. 6.

The second virial coefficient $B_{2}$ of our lattice model is given by ${ }^{5}$

$$
B_{2}=\frac{1}{2}\left[27-\frac{1}{6} \sum_{i=1}^{6} \sum_{j=1}^{6}\left(\exp \left(u_{i j}\right)-1\right)\right],
$$


where the first term inside the brackets comes from excluded-volume interactions and the second from the interactions between touching patches. The number 27 comes from the fact that each model protein excludes other proteins from a cube of $3 \times 3 \times 3$ lattice sites. Thus, in the high temperature limit $B_{2}=B_{2 h c}=27 / 2$. The sums over 24 orientations reduce to sums over six orientations as rotating either of the two molecules around the axis joining their centers does not change the energy. The factor in front of the double sum is a normalization factor of 1/36 times the six possible lattice sites that one molecule can occupy and be adjacent to the other molecule.

\section{STABILITY OF SOLUTIONS}

Unless $\epsilon$ is small, many of the $2^{6 n_{B}}$ model proteins strongly attract each other, leading to condensation, gelation, and possibly crystallization. By condensation we mean the formation of coexisting dilute and concentrated protein solutions, as have been studied extensively for the protein lysozyme. ${ }^{19,26,27}$ Only a fraction of the model proteins are viable in the sense that they are stable as single phase solutions. Clearly proteins cannot condense in vivo without severely impairing the organism's function.

The attractions affect the phase behavior through and can be measured by the second-virial coefficient. In the absence of attractions the second-virial coefficient is approximately four times the volume of a particle (assuming the particle is not too anisotropic). Attractions decrease its value until eventually the pressure does not increase monotonically but decreases over a range of densities due to the negative virial coefficient; a van der Waals loop forms. If we impose the constraint that the second virial coefficient be above a certain value, where we believe the pressure will be a monotonic function of density, we can quantify what fraction of our model proteins satisfy this constraint and so have solutions which are stable.

We insist that the reduced second virial coefficient satisfy $B_{2} / B_{2 h c} \geqslant-1$, in order for the protein to be viable. The fraction of proteins which are viable, according to this criterion, is denoted by $f_{v}$. It is determined by generating proteins at random, setting each bit to be 0 or 1 with equal probabilities, and finding the fraction with $B_{2} / B_{2 h c} \geqslant-1$. See the Appendix for further details of the computations. The value of $B_{2} / B_{2 h c}$ at the critical point, the highest point on the curve separating the one and two-phase regions of a phase transition into coexisting solutions, is typically a little less than -1 , unless the attraction is very anisotropic. For the canonical model, hard spheres plus a long-range attraction, the critical point occurs when $B_{2} / B_{2 h c}=-1.65$, and provided the attraction remains isotropic this value changes little even if the attraction is made quite short ranged. ${ }^{27}$ If the attraction is very anisotropic then $B_{2} / B_{2 h c}$ can (depending a little on the precise nature of the anisotropy) be much more negative at the critical point, ${ }^{28-30}$ but for simplicity we insist on $B_{2} / B_{2 h c}$ being above a fixed value for all our proteins, regardless of how anisotropic are their attractions. Crystallization out of not-too-concentrated solutions also requires as a minimum, attractions of about the strength required to make $B_{2} / B_{2 h c}$ around -1 . The propensity to crystallize de-

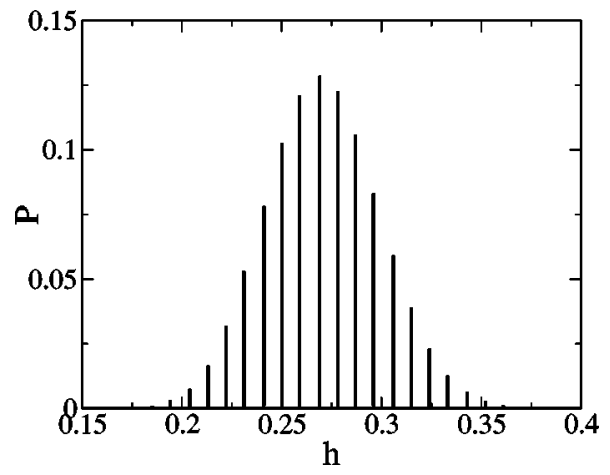

FIG. 7. The probability distribution function, $P$, for the fraction of bits hydrophobic, $h$. For $n_{B}=18$ and $\epsilon=2$.

pends on the details of the attraction, for work on the earlier version of this model with random values of the patch-patch attractions; see Ref. 5.

Results are shown, as a function of $\epsilon$, for $n_{B}=12$ and 18, in Fig. 4. As might have been expected, as $\epsilon$ increases, the fraction of viable proteins decreases exponentially, but note that even for $n_{B}=12$ and $\epsilon=2$, there are still $7.1 \times 10^{9}$ viable proteins, a very large number. Partly, what is happening is that as $\epsilon$ increases then fewer and fewer hydrophobic bits are allowed, and as the fraction of bits that are hydrophobic decreases, then the number of possible proteins decreases: there are many possible proteins with close to half their bits $0 \mathrm{~s}$ and half $1 s$, but only one with all its bits equal to 1. Partly, what happens is that correlations are introduced between the hydrophobic bits in the strings. The hydrophobic bits tend to avoid each other, e.g., if all six strings have all their bits from 1 to $n_{B} / 2$ (assuming $n_{B}$ is even) hydrophilic, then any or all of their bits from $n_{B} / 2+1$ to $n_{B}$ may be hydrophobic without there being any attractions. Thus here the hydrophobic bits avoid each other, in order to avoid the attractive interactions which make the second virial coefficient negative and thus violate our solubility condition.

We can measure both these effects by defining two quantities. The first is the mean fraction of bits which are 0 , are hydrophobic. Denoting this by $\langle h\rangle$, it is defined by

$$
\langle h\rangle=\frac{1}{6 n_{B}}\left\langle\sum_{i=1}^{6} \sum_{\alpha=1}^{n_{B}}\left(1-b_{\alpha}^{(i)}\right)\right\rangle .
$$

The average denoted by \langle\rangle is over proteins which satisfy our criterion for the stability of the solution. We use $h$ to denote both the fraction of bits in our model proteins that are hydrophobic, and the fraction of residues in real proteins that are hydrophobic. A measure of the correlation between the probability that a bit $\alpha$ is hydrophobic, and that the bit 1 $+n_{B}-\alpha$ with which it interacts is also hydrophobic is denoted by $\left\langle h h_{p}\right\rangle$, and is defined by 
$\left\langle h h_{p}\right\rangle=\frac{1}{36 n_{B}\langle h\rangle^{2}}\left\langle\sum_{i=1}^{6} \sum_{j=1}^{6} \sum_{\alpha=1}^{n_{B}}\left(1-b_{\alpha}^{(i)}\right)\left(1-b_{1+n_{B}-\alpha}^{(j)}\right)\right\rangle$.

We have plotted both quantities in Fig. 5. The model has $n_{B}=18$ bits and the quantities are plotted as a function of $\epsilon$. As $\epsilon$ increases, the fraction of bits which can be hydrophobic without the second virial coefficient becoming too negative decreases. Also, the anticorrelations between a bit being hydrophobic and the bit with which it interacts being also hydrophobic increases. If there were no correlation between the states of the 2 bits then $\left\langle h h_{p}\right\rangle=1$, which is true for $\epsilon=0$, but this function decreases as $\epsilon$ increases. If a bit is hydrophobic the bit with which it interacts is less likely to be hydrophobic. We have shown results just for $n_{B}=18$ but results for other numbers of bits are similar.

We only constrain the second virial coefficient to be above a certain value; we do not constrain its precise value. As the second virial coefficient is a function of the number of hydrophobic bits on its six faces and as this number is an integer between 0 and $n_{B}$, the second virial coefficient can only take one of a set of values, and so the probability density function for $B_{2} / B_{2 h c}$ is a set of delta functions. We have plotted these as spikes, with the height of each spike set to the probability that $B_{2} / B_{2 h c}$ has this value. We can see that the most likely values of the reduced second virial coefficient are near the minimum allowed value of -1 . This is simply because there are many more sets of strings with close to half the bits hydrophobic than there are with most of the bits hydrophilic, and the proteins with close to half the bits hydrophobic have very large and negative second virial coefficients. There is only one protein with all 108 bits hydrophilic but the number of proteins which have nine hydrophobic and nine hydrophilic bits on each face is $\left(18 ! / 9 !^{2}\right)^{6} \sim 10^{28}$. The probability distribution function for all possible proteins (including those with $B_{2} / B_{2 h c}<-1$ ) is sharply peaked at a value much less than 1 , for $n_{B}=18$ and $\epsilon=2$, and Fig. 6 shows just the high $B_{2}$ tail of this distribution.

The probability distribution function, again a sum of delta functions, of $h$ the fraction of hydrophobic bits, is plotted in Fig. 7. As with Fig. 6, $n_{B}=18$ and $\epsilon=2$. The distribution is peaked at $h$ a little above 0.25: the mean value $\langle h\rangle$ $=0.27$ and the standard deviation is 0.029 . As $h$ increases towards 0.5 then there are many more possible proteins but a rapidly increasing fraction of these are not soluble as a single phase according to our criterion. Thus there is a trade-off between the number of possible proteins and the fraction that are soluble. This trade-off results in most proteins having between $20 \%$ and $35 \%$ of their bits hydrophobic. This of course depends on $n_{B}$ and $\epsilon$. Increasing either one decreases $\langle h\rangle$ but the picture remains qualitatively the same.

Let us return to our results for adenylate kinases; Fig. 3. Although it should be borne in mind that many of adenylate kinases' hydrophobic amino acids will be in the center of the protein, not at its surface, we can still quantify the scatter in $h$ for the kinases, and compare it to the scatter in $h$ for the model proteins. But of course any comparison will be purely qualitative. The adenylate kinases have around 200 amino acids in total, of which about 100 are classified as hydrophobic. We can try to model the distribution functions for $h$, for both adenylate kinases and our model proteins, with

$$
h=n^{-1} \sum_{i=1}^{n} \zeta_{i}
$$

where for an adenylate kinase the sum is over all its amino acids in a protein, $n=M$, and for a model protein the sum is over the $n=6 n_{B}$ bits. The $\zeta_{i}$ are independent random variables which are 1 with probability $\langle h\rangle$ and zero otherwise. For adenylate kinases, see Fig. 3, we find that the standard deviation of $h$ is 0.040 , and Eq. (5) gives a standard deviation of 0.035 , only a little lower. To obtain the value of 0.040 we took the sum over 206 terms; 206 is the mean length of the adenylate kinases in Fig. 3. Taking all the proteins to be the same length will decrease the spread slightly. Note that we can predict the distribution of the proteins' hydrophobicity reasonably accurately using only the central limit theorem.

For our model proteins the standard deviation of $h$ is 0.029, while Eq. (5) predicts 0.043, which is rather larger but still comparable. Also, of course the shape of the distribution in Fig. 7 is quite close to Gaussian. Thus, the results for our model proteins are similar to those for real proteins, but as both are within a factor of 1.5 of a simple prediction based on assuming the hydrophobic amino acids/bits are randomly distributed, it is hard to draw definite conclusions from this. The distribution of net charges can also be modeled assuming that the charged amino acids are distributed at random. ${ }^{18,25}$

\section{CONCLUSION}

We started with the idea that globular proteins needed to be soluble to function, and that their interactions depended on their surfaces which in turn were sensitive to which types of amino acids were at the surfaces of proteins. Then we defined a very simple model of a protein, whose surfacemediated-interactions depended on the values of strings of bits. A mutation in a protein such as an adenylate kinase which substituted a hydrophobic amino acid at the surface for a hydrophilic one could then be modeled by flipping one of these bits. Within our model, and with the constraint that a solution of the model protein is stable; the second virial coefficient is rather variable, its probability distribution function is plotted in Fig. 6. The criterion for the solution to be stable as a single phase is taken to be that the reduced second virial coefficient $B_{2} / B_{2 h c} \geqslant-1$, which is enough for almost all fluids to be above their critical point. The condition that the protein solution be stable as a single phase is clearly a necessary condition, although in fact the second virial coefficient may be be more tightly constrained than this. Although the model used is simple, this variability does give credence to the idea that the variation in the fraction of hydrophobic amino acids in enzymes like adenylate kinase, see Fig. 3, gives rise to variability in the protein-protein interactions of these enzymes. In other words, that the second virial coefficients of $E$. coli's and $V$. cholerae's adenylate 
kinases may be significantly different, even though there is no obvious functional reason why their physical properties should differ. Unfortunately, virial coefficient measurements have not been performed for families of proteins. The variability is relevant to problems such as the purification and crystallization of proteins. The separation of one protein from all the others in an extract from a cell which might contain thousands of proteins relies on differences in physical properties, charge, surface stickiness, etc., between proteins.

The probability distribution function of the second-virial coefficient, Fig. 6, is just the high $B_{2}$ tail of the distribution of all proteins. The remainder of the distribution function is cut off by the requirement that $B_{2} / B_{2 h c} \geqslant-1$. This full distribution function has a peak at an $\epsilon$ dependent value of $B_{2}$; here well below -1 . Thus, without the cutoff at $B_{2} / B_{2 h c}$ $=-1$, the distribution function is similar to the Gaussian distribution function found for the earlier model in which the patch-patch interactions were taken to be random variables. ${ }^{5}$ If we had kept with the previous model of describing with random variables the patch-patch interactions, and required that $B_{2} / B_{2 h c} \geqslant-1$, then we would have obtained a distribution of second-virial coefficients similar to that in Fig. 7. In that sense a distribution like that in Fig. 7 is generic to any system where all model proteins except for those in a large $B_{2}$ tail are cut off. However, within the earlier, simpler, model there is no clear way to look at either mutations and hence evolution, or to compare with sequence data for real proteins, as we did when we compared Figs. 3 and 7.

Finally, many simplifying assumptions have been made in order to arrive at our model system. It is therefore appropriate to comment on how this work can be extended to include more of the features of proteins inside cells. Both the model and our simple criterion for viability can be improved. The model is rather crude, and our sharp division between proteins deemed soluble and those deemed insoluble, could be softened. Then the fitness of a protein would decline over some range of values of the second virial coefficient. Also, we did not impose a maximum on the second virial coefficient. If it is important to limit the osmotic pressure, values of the second virial coefficient which are too positive may also be undesirable. However, in terms of understanding the behavior of proteins in the complex crowded mixture of proteins that is the in vivo environment, perhaps the most important extensions of this work, is to multicomponent mixtures, and to include proteins which bind to each other. Inside cells thousands of different proteins are mixed together at a total protein concentration of around $20 \%$, and many proteins are not monomeric but are part of complexes. The model studied here is flexible enough to both generate thousands of different proteins and to permit selective binding between proteins. Work on both is ongoing.

\section{ACKNOWLEDGMENTS}

It is a pleasure to acknowledge that this work started with inspiring discussions with D. Frenkel. This work was supported by the Wellcome Trust (069242).

\section{APPENDIX: COMPUTATIONS}

We are principally interested in the fraction of proteins that are soluble according to our criterion, and the distribution functions and means of various properties of soluble proteins. The fraction of proteins with $B_{2} / B_{2 h c} \geqslant-1$ is determined by simply generating a very large number of proteins at random and finding the fraction that satisfy this requirement. The length of all runs are determined either by the requirement to obtain at least two significant figures or until longer runs produce almost identical plots. An exception is for $n_{B}=18$ and $\epsilon=2$ where due to the smallness of $f_{v}$, it was only possible to obtain 1 significant figure of accuracy. The distribution functions, means, etc., are obtained by starting with a soluble protein and generating a random walk in the space of soluble proteins. This is essentially no different from Metropolis Monte Carlo as applied to a system with a hard potential, e.g., a fluid of hard spheres, as our constraint $B_{2} / B_{2 h c} \geqslant-1$, is a hard constraint. The averages are then obtained over these random walks.

The algorithm samples "protein space," ${ }^{20}$ with each sequence a unique point in this space and two sequences neighbors if one of them can be transformed into the other by a single mutation. This protein space is vast for real proteins and still very large for our model; for our model it contains $2^{6 n_{B}}$ points. Note that all viable proteins are connected to all other viable proteins by an unbroken path of viable proteins and links between neighboring viable proteins. This is easy to see if we consider that $B_{2}$ always either increases or stays the same if we flip a hydrophobic bit. Thus, starting from any viable protein we can flip each of its hydrophobic bits to hydrophilic bits, one at a time, until we reach the protein with all $6 n_{B}$ bits hydrophilic. Each intermediate in this path must satisfy our solubility criterion as it is obtained from a protein which satisfies this criterion by flipping one or more hydrophobic bits. Thus we have proved that all viable proteins are connected to the protein with all hydrophilic bits, and so trivially all viable proteins are part of a connected network. This immediately implies that we can go from any one viable protein to any other via our Monte Carlo moves.

\footnotetext{
${ }^{1}$ The two proteins have SwISSPROT (Refs. 21 and 22) accession numbers Q9KTB7 and O66490. They are almost certainly descended from a common ancestral protein and have, despite their very different sequences, very similar three-dimensional structures. They are both part of the same family of proteins with accession number PS00113. See the PROSITE (Refs. 23 and 24) database of protein families.

${ }^{2}$ B. Alberts, D. Bray, J. Lewis, M. Raff, K. Roberts, and J. D. Watson, Molecular Biology of the Cell, 3rd ed. (Garland, New York, 1994).

${ }^{3}$ J. M. Berg, J. L. Tymoczko, and L. Stryer, Biochemistry, 5th ed. (Freeman, New York, 2002).

${ }^{4}$ D. Voet and J. G. Voet, Biochemistry, 2nd ed. (Wiley, New York, 1995). ${ }^{5}$ R. P. Sear, Europhys. Lett. 60, 938 (2002).

${ }^{6}$ Vibrio cholerae has 3785 proteins; see the proteome database (http:// www.ebi.ac.uk/proteome/). The genome of V. cholerae was sequenced, and hence the amino acid sequènces of its proteins determined by Heidelberg et al. (Ref. 31) at The Institute of Genomic Research (http:// www.tigr.org/)

${ }^{7}$ F. C. Neidhardt, "Chemical composition of E. coli, in E. coli and S. typhimurium," Cellular and Molecular Biology, edited by F. C. Neidhardt et al. (American Society for Microbiology, Washington DC, 1987).

${ }^{8}$ M. Kimura, Nature (London) 217, 624 (1968).

${ }^{9}$ J. L. King and T. H. Jukes, Science 164, 788 (1969).

${ }^{10}$ F. J. Ayala, Proc. Natl. Acad. Sci. U.S.A. 94, 7776 (1997).
} 
${ }^{11}$ U. Bastolla, M. Porto, H. E. Roman, and M. Vendruscolo, Phys. Rev. Lett. 89, 208101 (2002); J. Mol. Evol. 56, 243 (2003).

${ }^{12}$ U. Bastolla, M. Vendruscolo, and H. E. Roman, Eur. J. Biochem. 15, 385 (2000).

${ }^{13}$ E. J. Deeds, N. V. Dokholyan, and E. I. Shakhnovich, Biophys. J. 85, 2962 (2003).

${ }^{14}$ C. Tang, Physica A 288, 31 (2000).

${ }^{15}$ E. Bornberg-Bauer and H. S. Chan, Proc. Natl. Acad. Sci. U.S.A. 96, 10689 (1999).

${ }^{16}$ D. M. Taverna and R. A. Goldstein, J. Mol. Biol. 315, 479 (2002).

${ }^{17}$ P. G. Higgs, Q. Rev. Biophys. 33, 199 (2000).

${ }^{18}$ R. P. Sear, J. Chem. Phys. 118, 5157 (2003).

${ }^{19}$ D. F. Rosenbaum, A. Kulkarni, S. Ramakrishnan, and C. F. Zukoski, J. Chem. Phys. 111, 9882 (1999).

${ }^{20}$ J. Maynard-Smith, Nature (London) 225, 563 (1970).
${ }^{21}$ SWISSPROT is a curated protein sequence database; see http:// www.expasy.org/sprot/.

${ }^{22}$ B. Boeckmann, Nucleic Acids Res. 31, 365 (2003).

${ }^{23}$ PROSITE is a database of protein families; see http://www.expasy.org/ prosite/.

${ }^{24}$ C. J. A. Sigrist et al., Brief Bioinform. 3, 265 (2002).

${ }^{25}$ R. P. Sear, unpublished work.

${ }^{26}$ M. Muschol and F. Rosenberger, J. Chem. Phys. 107, 1953 (1997).

${ }^{27}$ G. A. Vliegenthart and H. N. W. Lekkerkerker, J. Chem. Phys. 112, 5364 (2000).

${ }^{28}$ R. P. Sear, J. Chem. Phys. 111, 4800 (1999).

${ }^{29}$ R. A. Curtis, H. W. Blanch, and J. M. Prausnitz, J. Phys. Chem. B 105, 2445 (2001)

${ }^{30}$ N. Kern and D. Frenkel, J. Chem. Phys. 118, 9882 (2003).

${ }^{31}$ J. F. Heidelberg et al., Nature (London) 406, 477 (2000). 